\title{
Capacity assessment of the health laboratory system in two resource- limited provinces in China
}

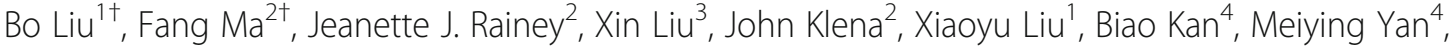 \\ Dingming Wang ${ }^{5}$, Yan Zhou ${ }^{6}$, Guangpeng Tang ${ }^{5}$, Mingliu Wang ${ }^{6}$ and Chihong Zhao ${ }^{{ }^{*}}$
}

\begin{abstract}
Background: Strong laboratory capacity is essential for detecting and responding to emerging and re-emerging global health threats. We conducted a quantitative laboratory assessment during 2014-2015 in two resource-limited provinces in southern China, Guangxi and Guizhou in order to guide strategies for strengthening core capacities as required by the International Health Regulations (IHR 2005).

Methods: We selected 28 public health and clinical laboratories from the provincial, prefecture and county levels through a quasi-random sampling approach. The 11-module World Health Organization (WHO) laboratory assessment tool was adapted to the local context in China. At each laboratory, modules were scored $0-100 \%$ through a combination of paper surveys, in-person interviews, and visual inspections. We defined module scores as strong ( $>=85 \%)$, good (70-84\%), weak (50-69\%), and very weak ( $<50 \%)$. We estimated overall capacity and compared module scores across the provincial, prefecture, and county levels.

Results: Overall, laboratories in both provinces received strong or good scores for 10 of the 11 modules. These findings were primarily driven by strong and good scores from the two provincial level laboratories; prefecture and county laboratories were strong or good for only 8 and 6 modules, respectively. County laboratories received weak scores in 4 modules. The module, 'Public Health Functions' (e.g., surveillance and reporting practices) lagged far behind all other modules (mean score $=46 \%$ ) across all three administrative levels. Findings across the two provinces were similar.

Conclusions: Laboratories in Guangxi and Guizhou are generally performing well in laboratory capacity as required by IHR. However, we recommend targeted interventions particularly for county-level laboratories, where we identified a number of gaps. Given the importance of surveillance and reporting, addressing gaps in public health functions is likely to have the greatest positive impact for IHR requirements. The quantitative WHO laboratory assessment tool was useful in identifying both comparative strengths and weaknesses. However, prior to future assessments, the tool may need to be aligned with the new WHO IHR monitoring and evaluation framework.
\end{abstract}

Keywords: International health regulations, Laboratory assessment, Public health laboratory, Clinical laboratory, Resource-limited, Guangxi, Guizhou, China

\footnotetext{
* Correspondence: zhaoch@chinacdc.cn

Bo Liu and Fang Ma are equal contributors as first authors.

${ }^{1}$ Office of Laboratory Management, Chinese Center for Disease Control and

Prevention, Room 335, 155 Changbai Road, Changping District, Beijing

102206, People's Republic of China

Full list of author information is available at the end of the article
}

(c) The Author(s). 2019 Open Access This article is distributed under the terms of the Creative Commons Attribution 4.0 International License (http://creativecommons.org/licenses/by/4.0/), which permits unrestricted use, distribution, and reproduction in any medium, provided you give appropriate credit to the original author(s) and the source, provide a link to the Creative Commons license, and indicate if changes were made. The Creative Commons Public Domain Dedication waiver (http://creativecommons.org/publicdomain/zero/1.0/) applies to the data made available in this article, unless otherwise stated. 


\section{Background}

The International Health Regulations (IHR, 2005) published by the World Health Organization (WHO) describe the minimum core capacities required to detect, assess, report, and respond to public health emergencies of international concern [1]. China follows the IHR in responding to common threats such as seasonal Influenza A as well as transmission of novel infections such as Severe Acute Respiratory Disease Syndrome (SARS) [2]. Strong laboratory capacity is essential for detecting and responding to public health threats as outlined in the Regulations.

In China, front-line detection and response responsibilities rely on the provincial-, prefecture-, and county-level Centers for Disease Control. At each administrative level, public health laboratories collaborate with hospital-based clinical laboratories to investigate suspected cases of emerging and re-emerging infections. County- and prefecturelevel laboratories conduct field-based epidemiological investigations and perform initial specimen collection and testing, and provincial-level laboratories provide molecular testing and sub-typing for further identification purposes and coordinate surveillance activities.

Maintaining laboratory capacity across each administrative level and ensuring collaboration between the public health and clinical laboratories are key for full IHR adherence. Since maintaining capacity and ensuring collaboration could be more challenging in resource-limited areas, we conducted a systematic laboratory assessment in two resource-limited provinces in southern China, Guangxi and Guizhou, using the Laboratory Assessment Tool (LAT) developed by WHO. To the best of our knowledge, this is the first application and publication of quantitative scores for assessing laboratory capacity in multiple provinces of China. Findings from this assessment will be used to generate data-drive evidence on current laboratory capacity and need for specific interventions in Guangxi and Guizhou Provinces.

\section{Methods}

\section{Assessment provinces and laboratory selection}

We conducted this assessment in two resource-limited provinces of China, Guizhou and Guangxi. In 2014 the populations of Guangxi and Guizhou were 47,540,000 and 35,080,000, respectively. The average annual Gross Domestic Product (GDP) for these provinces is approximately $\$ 194.7$ billion [3], substantially lower than the national average of $\$ 295.9$ billion [4]. The provinces are administratively divided into prefectures and counties; counties are further divided into townships. Outbreaks of Salmonella typhi and paratyphi are common in Guangxi and Guizhou [5, 6]. Both provinces have detected Avian Influenza A H7N9 [7, 8].
We used a stratified random sampling approach to select two prefectures in Guizhou (22.2\% of all prefectures) and two in Guangxi (14.3\% of all prefectures). Then from each of these four prefectures, we randomly selected two counties (26.7 and $19.0 \%$ of the counties in the two selected prefectures in Guizhou and Guangxi, respectively). At each administrative level, laboratories from both public health and clinical sectors were selected. The selection approach resulted in a total of 14 laboratories for each province (provincial level $[n=2$ laboratories], prefecture $[n=4]$, and county $[n=8])$, including 7 hospital-based clinical laboratories. Public laboratories (PHLs) and clinical laboratories (CLs) are managed by separate agencies at each administrative level.

\section{Assessment tool}

The WHO LAT is a generic document containing a number of modules and indicators that can be adapted by public health officials to evaluate specific core laboratory capacities and capabilities at the national or local levels. For this assessment, we selected 11 modules from the WHO LAT (Table 1). These modules were adapted to the local context (i.e., modified wording to improve interpretation), translated into Chinese and pilot-tested prior to initiating the assessment. Each of the 11 modules included multiple indicators (ranging from 3 to 10 per module), quantitatively scored between 0 and 100\% [9]. The module score was calculated as the average across the module's indicators. We generated the overall laboratory assessment score as the average of the 11 module scores. Standard deviations were included to measure the spread or distribution around each calculated average.

\section{Data collection and analysis}

We obtained initial information through assessment surveys mailed to the laboratory manager at each selected laboratory. Two teams of two members each conducted on-site visits to verify these self-administered surveys as well as to conduct laboratory inspections. Team members received training on the use of the assessment tool and interview techniques by the China CDC staff. The assessments were conducted from July to November 2014 in both provinces. Verification and inspection data were documented on standardized paper forms.

We double-entered all survey and verification data into an Excel database and reconciled identified inconsistencies and missing data prior to conducting the descriptive analysis. We compared and contrasted assessment findings across the three administrative levels as well as between public health laboratories (PHLs) and clinical laboratories (CLs). We categorized module scores as strong $(>=85 \%)$, good (70-84\%), weak (50-69\%) and very weak $(<50 \%)$. 
Table 1 Assessment modules included in the adapted WHO Laboratory Assessment Tool used in Guangxi and Guizhou Provinces, China, 2014

\begin{tabular}{ll}
\hline Modules & Laboratory Capacities \\
Organization \& management & Internal \& external communication, budget, licensing/supervision/accreditation \\
Documents & Document control system, quality procedures, biosafety documents \\
Specimen collection, handling \& transport & Specimen collection, handling, referral/transport \\
Data \& information management & Test results and reports, data analysis and statistics, data security \& confidentiality, it and \\
laboratory information management system (LIMS)
\end{tabular}

We examined and described the individual indicators for modules where the scores for any administrative level was $<70 \%$. Data were entered into SPSS (version 16.0, New York, USA) for analysis, assuming quasi-random sampling (i.e., results were not adjusted for varying selection probabilities within and across the two provinces).

No personal identifying information from patients or specimens was obtained during the assessment. China CDC and Guangxi and Guizhou Provincial CDCs approved the assessment as non-research. The assessment was also determined to be non-research by the Human
Subjects Research Determination Process at the United States Centers for Disease Control and Prevention and therefore exempt from IRB review.

\section{Results \\ Overall assessment scores}

On average, the laboratories selected for this assessment received an overall good $(75.7 \%)$ score across the 11 modules (Table 2). Provincial laboratories received the highest overall score $(79.6 \%)$ followed by prefecture (77.8\%) and county (73.7\%) laboratories. Among the 11

Table 2 Aggregated performance scores on 11 assessment modules for 28 laboratories, categorized by administrative level (provincial, prefecture, and county) and laboratory sector (Public Health - PH, and Clinical - CL), in Guangxi and Guizhou Provinces, China, 2014

\begin{tabular}{|c|c|c|c|c|c|c|c|c|c|c|}
\hline \multirow[t]{2}{*}{ Assessment modules } & \multirow{2}{*}{$\begin{array}{l}\text { Average } \\
(\%)\end{array}$} & \multicolumn{3}{|c|}{ Provincial (\%) } & \multicolumn{3}{|c|}{ Prefecture (\%) } & \multicolumn{3}{|l|}{ County (\%) } \\
\hline & & All & $\mathrm{PH}$ & $\mathrm{CL}$ & All & $\mathrm{PH}$ & $\mathrm{CL}$ & All & $\mathrm{PH}$ & $\mathrm{CL}$ \\
\hline Average & 75.7 & $79.6(13.5)$ & $81.5(15.3)$ & $77.6(17.3)$ & $77.8(7.6)$ & $75.9(5.3)$ & $79.8(9.9)$ & 73.7 (14.6) & $72.8(14.8)$ & $74.6(15.5)$ \\
\hline $\begin{array}{l}\text { Organization \& } \\
\text { management }\end{array}$ & 71.4 & $77.6(12.4)$ & 74.3 (18.6) & $80.9(8.4)$ & 74.7 (14.5) & $66.1(5.8)$ & $83.3(16.1)$ & $68.1(18.3)$ & $67.5(25.2)$ & $68.8(8.9)$ \\
\hline Documents & 75.4 & $87.7(20)$ & $97.7(3.2)$ & $77.6(27.9)$ & $83.6(15.3)$ & $88.3(9.4)$ & 78.9 (19.9) & $68.2(27.8)$ & $74.1(30.5)$ & $62.3(25.4)$ \\
\hline $\begin{array}{l}\text { Specimen collection, } \\
\text { handling \& transport }\end{array}$ & 85.3 & $80.4(11.2)$ & $77.4(15.2)$ & $83.4(10.6)$ & $89.1(6.2)$ & $87.1(7.4)$ & $91(5.1)$ & $84.6(14)$ & $87(10.7)$ & $82.3(17.1)$ \\
\hline $\begin{array}{l}\text { Data \& information } \\
\text { management }\end{array}$ & 81.6 & $75.8(17)$ & $71.3(24.7)$ & $80.3(13.3)$ & $82.6(12.3)$ & $82.2(16.2)$ & $83(9.6)$ & $82.6(14.6)$ & $85.1(14.9)$ & 80.1 (14.8) \\
\hline $\begin{array}{l}\text { Consumables \& } \\
\text { reagents }\end{array}$ & 83.3 & $85.5(12.4)$ & $85.4(15.2)$ & $85.7(15.2)$ & $83.8(9.6)$ & $79.6(7.1)$ & $88.1(10.8)$ & $82.5(15.7)$ & $80.3(12.2)$ & $84.6(19.2)$ \\
\hline Equipment & 87.6 & 84.5 (16.3) & $75(20.5)$ & $94(4.8)$ & $90.9(11)$ & 89.5 (13.8) & $92.3(9.4)$ & $86.8(18.5)$ & $88.6(14.1)$ & $84.9(23)$ \\
\hline $\begin{array}{l}\text { Laboratory testing } \\
\text { performance }\end{array}$ & 71.3 & 70.7 (41.9) & $87.2(2.7)$ & $54.3(64.7)$ & $68.4(22.6)$ & $64.9(27.9)$ & 71.8 (19.5) & $72.9(23.4)$ & $73.8(24.9)$ & 72 (23.6) \\
\hline Facilities & 71.5 & $78.4(22)$ & $70.5(34.7)$ & $86.4(0)$ & $75.1(21.1)$ & $63.6(24.5)$ & $86.5(9.7)$ & $67.9(18.9)$ & 69 (19.6) & $66.8(19.6)$ \\
\hline Human resources & 79.5 & $88.8(6.3)$ & $85(7.1)$ & $92.5(3.5)$ & $80(16.3)$ & $68.8(13.1)$ & $91.3(10.3)$ & $76.9(16.2)$ & $71.9(14.6)$ & $81.9(17.1)$ \\
\hline Biorisk management & 75.9 & $89.6(10.9)$ & $87.1(14.4)$ & $92.1(11.2)$ & $81.1(18.8)$ & $72.9(23.2)$ & $89.3(10.6)$ & $69.9(29.9)$ & $57.5(32.8)$ & $82.3(22)$ \\
\hline Public health functions & 47.6 & $56(40.7)$ & $85.1(11.6)$ & $26.9(38.1)$ & $46.8(31.8)$ & $71.7(17.7)$ & $21.9(19.8)$ & $45.7(27.8)$ & $46.5(26.5)$ & $44.6(32)$ \\
\hline
\end{tabular}


modules, laboratories were overall strong $(\geq 85 \%)$ in 'Equipment' and 'Specimen Collection' and good ( $\geq 70 \%)$ in 'Organization and Management', 'Documents', 'Specimen Handling and Transport', 'Data and Information Management', 'Consumables and Reagents', 'Laboratory Testing Performance,' 'Facilities', 'Human Resources' and 'Biorisk Management'. Laboratories were weak or very weak $(\leq 50 \%)$ in 'Public Health Functions'. These findings were generally similar across the two provinces.

\section{Assessment scores by administrative level}

At least one administrative level scored weak, or $<70 \%$, for the following six modules, 'Organization and Management,' 'Documents', 'Laboratory Testing Performance', 'Facilities', 'Biorisk Management' and 'Public Health Functions'. This finding was primarily driven by weak county-level scores. The exception was for 'Laboratory Testing Performance' where only prefecture level laboratories scored $<70 \%$. To better understand the 'causes' of these weak scores, the individual indicators for these six modules are described below (Figs. 1, 2, 3, 4, 5, 6). We describe differences in module scores between public health and clinical laboratories separately below.

\section{Organization and management}

The 'Organization and Management' module scores reflect the average across five separate indicators (Fig. 1). County-level laboratories scored weak on the 'Budget' (51\%) and 'Qualification and Accreditation' (68\%) indicators, substantially lower than the other administrative levels. Prefecture laboratories scored weak on 'Qualification and Accreditation' (59\%), but interestingly scored higher than provincial laboratories for 'External Communication' and 'Budget'.

\section{Documents}

County-level laboratories scored lower than prefectureand provincial-level laboratories for each of the three indicators that comprise the 'Documents' module (Fig. 2). Although county-level laboratories scored good on 'Biosafety Documents', the overall weak module ranking was due to weak scores for 'Document Control System' (65\%) and 'Quality Procedures' (65\%).

\section{Laboratory testing performance}

Figure 3 shows the variability across the three administrative levels for each of the four Laboratory Testing Performance indicator scores. The overall weak module score for prefecture level laboratories was due to weak scores in Virology (62\%) and Parasitology (50\%); however, these same laboratories earned strong scores for the 'Food Testing' indicator. Interestingly, county-level laboratories scored higher for all four indicators than their provincial and prefecture counterparts.

\section{Facilities}

The 'Facilities' module scores reflect the average across two indicators (Fig. 4.). As with the 'Documents' module, county-level laboratories scored $<70 \%$ for these indicators, lower than both prefecture and provincial laboratories.

\section{Biorisk management}

Similar to the 'Documents' module, county-level laboratories scored lower than prefecture- and provincial-level laboratories for each of the three indicators that comprised the 'Biorisk Management' module. Laboratories at all levels scored strong or good on 'Implementation and Operation' indicator, while scores are weak on 'Biorisk Assessment and Control' indicator.

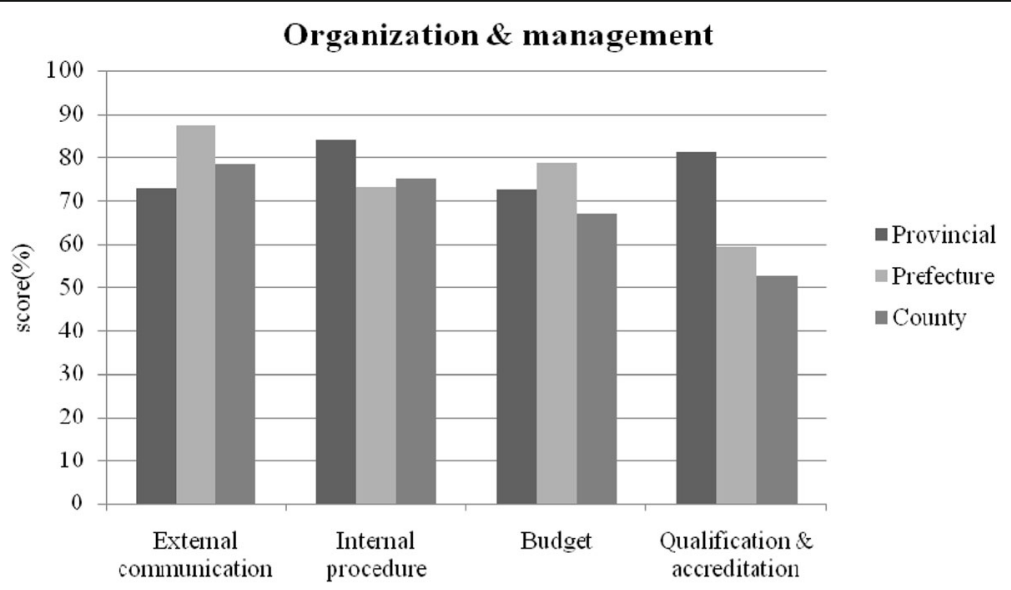

Fig. 1 Performance scores of the 'Organization and Management' module for the 28 laboratories by administrative levels (county, prefecture and province), Guangxi and Guizhou Provinces, China, 2014. Note: The average module score at the provincial-level $=77.6 \%$, prefectural-level $=74.7 \%$ and, county-level $=68.1 \%$ 


\section{Documents}

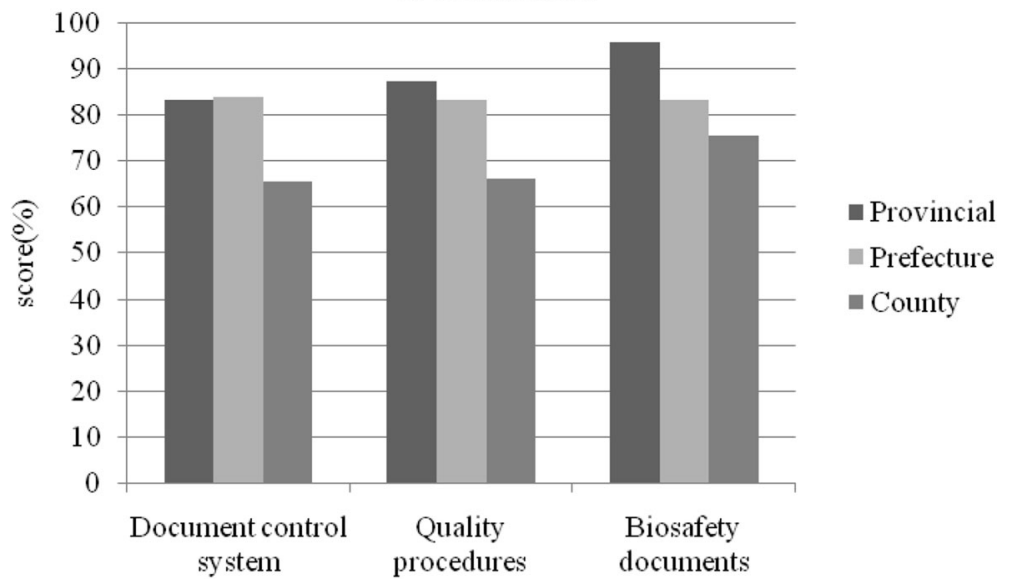

Fig. 2 Performance scores of the 'Documents' module for the 28 laboratories by administrative levels (county, prefecture and province), Guangxi and Guizhou Provinces, China, 2014. Note: The average module score at the provincial-level $=87.7 \%$, prefectural-level $=83.6 \%$, and county-level $=68.2 \%$

\section{Public health functions}

Performance on the 'Public Health Functions' module was considered weak or very weak across the three administrative levels. This finding reflects low scores for each of the three 'Public Health Function' indicators (Fig. 6). Prefecture- and county-level laboratories performed very weak in 'Surveillance and Response' and 'Public Health Reporting' (40 and 30\%, respectively). Provincial laboratories scored very weak (45\%) in 'Specimens for Public Health Purpose'.

\section{Module performance for public health and clinical laboratories}

Hospital-based clinical laboratories and public health laboratories generally received similar scores for seven of the 11 modules included in this assessment (Table 2). Differences were observed for 'Organization and Management',
'Facilities', and 'Biorisk Management' modules where hospital-based clinical laboratories scored higher than their public health counterparts at each administrative level. At the same time, CLs were substantially weaker than PHLs in 'Public Health Functions', greatly impacting the overall score for this module, particularly at the provincial and prefecture levels. Most noteworthy are the lower scores among CLs for 'Public Health Functions'. We have included figures comparing these module indicators between public health and clinical laboratories in the Additional files 1, 2, 3 and 4.

\section{Discussion}

According to this quantitative laboratory assessment in multiple provinces of China on laboratory capacity as required by IHR, laboratories in Guangxi and Guizhou Provinces are generally performing well for most of the LAT modules. Two modules, 'Specimen Collection,

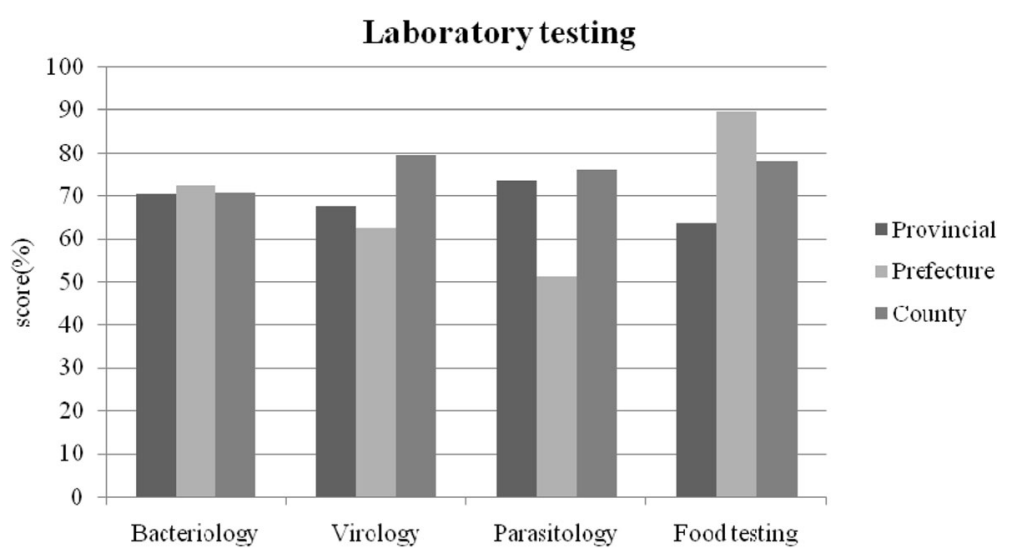

Fig. 3 Performance scores of the 'Laboratory Testing' module for the 28 laboratories by administrative levels (county, prefecture and province), Guangxi and Guizhou Provinces, China, 2014. Note: The average module score at the provincial-level $=70.7 \%$, prefectural-level $=68.4 \%$, and county-level $=72.9 \%$ 


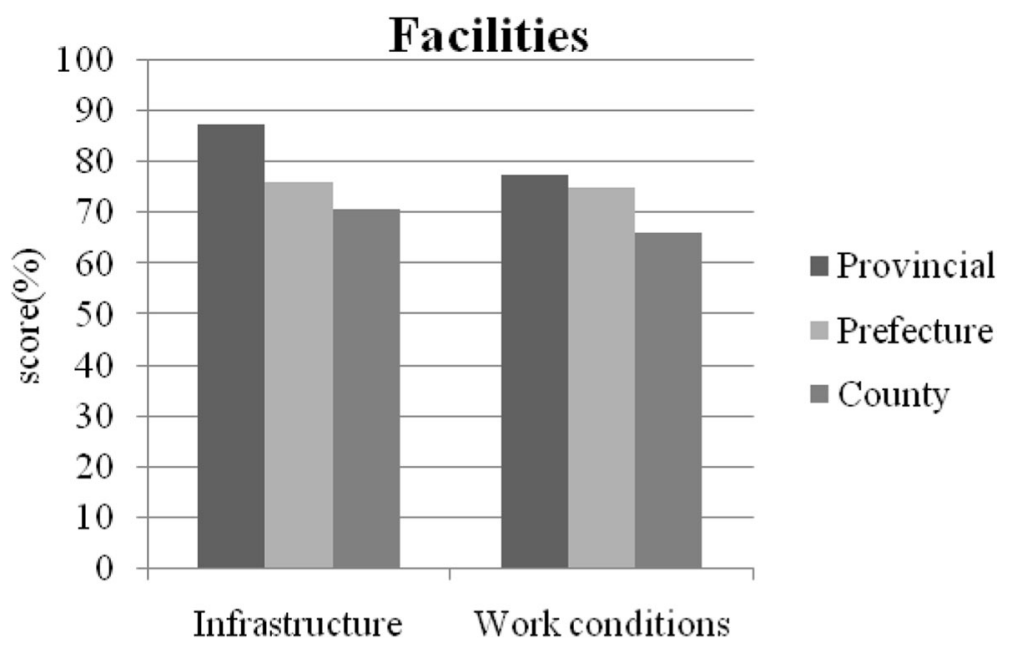

Fig. 4 Performance scores of the 'Facilities' module for the 28 laboratories by administrative levels (county, prefecture and province), Guangxi and Guizhou Provinces, China, 2014. Note: The average module score at the provincial-level $=78.4 \%$, prefectural-level $=75.1 \%$, and county-level $=67.9 \%$

Handling and Transport' and 'Equipment' received strong scores and six other modules received good scores. These findings likely reflect the long-term commitment of national and local Chinese leaders for laboratory capacity and system development. However, our assessment also identified a number of substantial performance gaps, particularly for the module 'Public Health Functions', where clinical laboratories were particularly weak in surveillance and response capacities. Additionally, county-level laboratories generally scored lower than prefecture and provincial laboratories on a number of modules.
The weak or very weak performance scores for 'Public Health Functions' were consistent across provincial-, prefecture-, and county-level laboratories and lagged far behind the other 10 capacities required by IHR. These scores were primarily driven by low surveillance and response capacity among clinical laboratories. Hospitalbased CLs play an essential role in fulfilling public health functions, particularly for specimen collection, clinical diagnostics and specimen referral, as well as reporting diagnostic test results in an accurate and timely manner. Improving collaboration between laboratories across the two sectors and sharing testing and confirmatory test

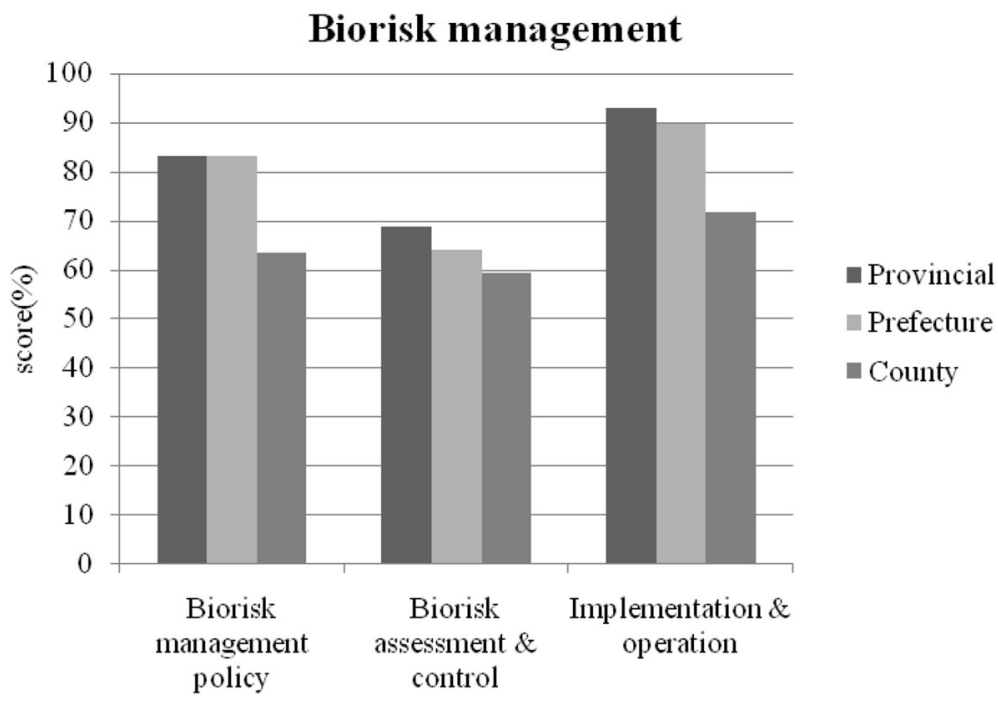

Fig. 5 Performance scores of the 'Biorisk Management' module for the 28 laboratories by administrative levels (county, prefecture and province), Guangxi and Guizhou Provinces, China, 2014. Note: The average module score at the provincial-level $=89.6 \%$, prefectural-level $=81.1 \%$, and county-level $=69.9 \%$ 


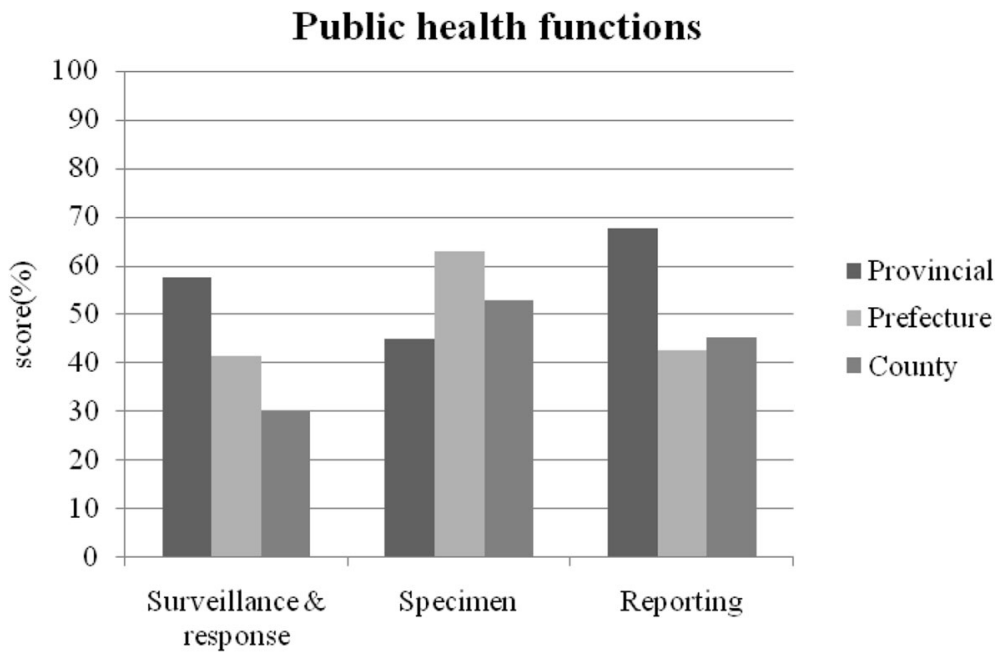

Fig. 6 Performance scores of the 'Public Health Functions' module for the 28 laboratories by administrative levels (county, prefecture and province), Guangxi and Guizhou Provinces, China, 2014. Note: The average module score at the provincial-level $=56 \%$, prefectural-level $=46.8 \%$, and county-level $=45.7 \%$

results could strengthen both laboratories, especially when confronted with emerging and re-emerging diseases. Since CLs scored high on 'Biorisk Management' [10] at all administrative levels, such collaboration could also reinforce implementation of bio-risk control measures among PHLs through cross-sector trainings and meetings.

Under the guidance of the Law of Infectious Diseases Prevention and Treatment [11], the National Health and Family Planning Commission (formerly called Ministry of Health) in China currently supports a number of disease-specific surveillance systems that cover field investigations, diagnostics, and reporting for individual etiologies [12]. Inadvertently, this vertical approach for many different diseases and syndromes could have negatively impacted capacity for implementing public health functions in both PHLs and CLs [12]. This is likely most pronounced in provinces with limited laboratory resources. Integrating disease-specific systems into surveillance and laboratory networks could help maximize available resources as well as strengthen engagement of all laboratories in surveillance and response activities.

The use of a quantitative scoring system allowed us to identify critical differences in laboratory capacity for a number of modules across provincial, prefecture, and county-level laboratories. The most noteworthy differences were observed for 'Organization and Management', 'Documents', 'Facilities', 'Laboratory testing' and 'Biorisk Management'. With the exception of 'Laboratory Testing, performance scores for each of these modules were typically highest for provincial laboratories, followed by prefecture laboratories and lowest for county-level laboratories. This finding may be partially explained by financial constraints often experienced by county-level laboratories [13]. These constraints can negatively affect staff recruitment, personnel training and capacity development, as reflected in the weak county-level scores for 'Facilities' and 'Human Resources'.

Since county- as well as prefecture-level laboratories are responsible for field-based epidemiological investigations and perform initial specimen collection and testing, the weak scores for these modules need to be addressed. Available tools such as the IHR implementation roadmap [14] to guide laboratory management and quality improvement could be used to address gaps across all levels. For instance, we can match an assessed capacity, such as 'Documents', with the corresponding phase of the roadmap and implement interventions to guide improvements in this capacity area. Interventions can include developing and improving procedures for testing, increasing availability and quality of laboratory equipment, strengthening inventory management as well as biosafety practices. Checklists can be used to verify completion of each activity. Additionally, these tools, along with support from provincial laboratories, could help prefecture laboratories meet accreditation requirements and competences [1].

Laboratories included in this assessment scored an average of $71 \%$ for the module 'Laboratory Testing Performance'. National guidance was published in 2004 describing the categories of tests that PHLs at each administrative level should be able to detect [15]. This guidance includes testing for diseases caused by bacteria, viruses, parasites and Rickettsia. It also includes testing conducted in preventive and check-up settings, such as for non-communicable diseases as well as chemicals and 
hazards in water, cosmetics, and the environment. According to the guidance document, PHLs at the provincial, prefecture, and county levels should be able to perform at least 433, 359 and 179 pathogen-specific diagnostic tests, respectively. Our assessment scored capacity according to this guidance and assessed 82 categories of tests for each administrative level, which is approximately one-fifth, one-fourth, and one-half of the required categories of tests for provincial, prefectural and county levels. Additionally, we did not compare testing types or accuracy for pathogen detection. This may partially explain the higher scores for county-level laboratories when compared to the provincial and prefecture laboratories included in this assessment.

Based on our assessment, national guidance appears to be generally helpful for PHLs. A similar guidance document addressing the specific challenges for CLs along with a training component aimed at increasing capacity in Public Health Functions could be beneficial. Such guidance and training will require closer collaboration and long-term commitments by the agencies managing the separate laboratory sectors.

We collaborated with international, national, and local public health staff and hospital administrators to design and conduct this laboratory assessment. This collaborative approach increased the likelihood that the assessment would be successfully implemented at each administrative level and across the two sectors. We were able to customize the LAT to the China laboratory structure; however, the resulting data may not applicable or comparable to other types of laboratories or to other countries. The tool allowed us to use the same quantitative score system while modifying the cut-offs for strong, good, and weak as laboratory capacity improves over time. WHO recently launched a new IHR monitoring and evaluation framework, including the Joint External Evaluation (JEE) tool [16]. This framework will combine self-evaluation, peer review and voluntary external evaluations for assessing IHR laboratory core capacities. Prior to future assessments, the LAT may need to be aligned with this new WHO IHR monitoring and evaluation framework.

We collected self-reported data from laboratory managers, and several different team members conducted the field verification and inspection visits for all modules to minimize possible bias. Project leads trained staff at each participating laboratory on the use of the self-assessment tool as well as members of the field visit teams prior to data collection. Nevertheless, PHL and CL staff could have had different interpretations of the survey questions, such as on biorisk management, due to their work focus. Additionally, we elected to use a multi-stage sampling approach that reduced travel time and resources required to complete the assessment (i.e., visiting county level laboratories located in only two different prefectures in each Province). Consequently, our findings, particularly at county-level, may not be representative for other laboratories in the two provinces. According to our local partners differences between prefectures are likely to be minimal.

\section{Conclusion}

We were able to successfully implement a quantitative assessment of laboratory capacity in two resource-limited provinces in China. From this assessment, laboratories are generally performing well in laboratory capacity as required by IHR, with the exception of 'Public Health Functions. Addressing gaps identified in this module, particularly in surveillance and response capacities, are likely to have the greatest positive impact for global health security and IHR requirements. We also recommend targeted interventions for county-level laboratories, where capacity was weak for a number of modules. Opportunities to strengthen collaboration between public health and clinical sectors could also be beneficial. Findings from this assessment can serve as a baseline to evaluate the impact of these targeted interventions.

\section{Additional files}

\begin{abstract}
Additional file 1: Performance scores combined across three administrative levels (county, prefecture and province) for the 11 assessment modules for the 28 laboratories by laboratory type (public health and clinical), Guangxi and Guizhou Provinces, China, 2014. (DOC $95 \mathrm{~kb}$ )

Additional file 2: Provincial-level performance scores for the 11 assessment modules for the 28 laboratories by laboratory type (public health and clinical), Guangxi and Guizhou Provinces, China, 2014. (DOC $93 \mathrm{~kb}$ )

Additional file 3: Prefecture-level performance scores for the 11 assessment modules for the 28 laboratories by laboratory type (public health and clinical), Guangxi and Guizhou Provinces, China 2014. (DOC 90 kb)

Additional file 4: County-level performance scores for the 11 assessment modules for the 28 laboratories by laboratory type (public health and clinical), Guangxi and Guizhou Provinces, China 2014. (DOC 89 kb)
\end{abstract}

\section{Abbreviations \\ CDC: Centers for Disease Control and Prevention; CLs: Clinical laboratories; IHR: International Health Regulations; LAT: Laboratory Assessment Tool;} PHLs: Public health laboratories; WHO: World Health Organization

\section{Acknowledgements}

We thank staff at the 28 participating laboratories in Guizhou and Guangxi Provinces for their support in data collection. We also thank Dr. Hong Chen and Mr. Kai Kang of the Program Management Office with China CDC for assisting in assessment implementation.

Funding

The assessment was funded by the Centers for Disease Control and Prevention under the Cooperative Agreement Number GH12-1247/1U2GGH000961. And the publication of this article was fully funded by China CDC. 


\section{Availability of data and materials}

According to study approval from China CDC, data and materials concerning the laboratory capacities of each participating laboratory will be kept away from public access.

\section{About this supplement}

This article has been published as part of BMC Public Health Volume 19 Supplement 3, 2019: 10th anniversary of the Centers for Disease Control and Prevention - Global Disease Detection program. The full contents of the supplement are available online at https://bmcpublichealth.biomedcentral. com/articles/supplements/volume-19-supplement-3.

\section{Authors' contributions}

Among the contributing authors of this article, BL was engaged in study design, field survey, data processing and analysis and paper writing; FM, JK and XL actively participated in study design and field survey; JR was deeply involved in data analysis and paper writing; XYL was involved in data analysis; BK and MY participated in study design and field survey; DW and GT were responsible for local organization of the field trips in Guizhou Province of China; YZ and MW were responsible for local organization of the field trips in Guangxi Province of China; CZ was principle investigator for the whole research. All authors of this article have read and approved the final manuscript.

\section{Ethics approval and consent to participate}

This study was approved by both China CDC and US CDC. Local laboratory staff involved in the field surveys were all informed that the data to be collected and analyzed in all laboratory capacity modules would involve no personal identity and private information. Consents from interviewees of the participating local laboratories were obtained before the field surveys.

\section{Consent for publication}

Not applicable.

\section{Competing interests}

There's no competing interests involved in this study for any of the authors of this article.

\section{Publisher's Note}

Springer Nature remains neutral with regard to jurisdictional claims in published maps and institutional affiliations.

\section{Author details \\ 'Office of Laboratory Management, Chinese Center for Disease Control and Prevention, Room 335, 155 Changbai Road, Changping District, Beijing 102206, People's Republic of China. ${ }^{2}$ Emerging and Infectious Disease Program, Centers for Disease Control and Prevention, Beijing 100600, China. ${ }^{3}$ Division of Global Health Protection, Centers for Disease Control and Prevention, Atlanta, GA 30329-4027, USA. ${ }^{4}$ National Institute for Communicable Disease Control and Prevention (ICDC), Chinese Center for Disease Control and Prevention, Beijing 102206, China. ${ }^{5}$ Guizhou Center for Disease Control and Prevention, Guizhou Province 550004, China. ${ }^{6} \mathrm{Guangxi}$ Center for Disease Control and Prevention, Guangxi Province 530028, China.}

Published: 10 May 2019

\section{References}

1. World Health Organization. International health regulations (2005), 3rd Available at http://www.who.int/ihr/publications/9789241580496/en/. Last accessed on 2 Dec. 2016.

2. Editorial (no author). From SARS to H7N9: will history repeat itself? Lancet. 2013;381:1333.

3. National Bureau of Statistics of China. Regional GDP in 2014. Available at http://data.stats.gov.cn/easyquery.htm?cn=E0103. Last accessed on 2 Dec 2016

4. National Bureau of Statistics of China. National GDP in 2014. Available at http://data.stats.gov.cn/search.htm?s=\%E5\%90\%84\%E7\%9C\%81GDP. Last accessed on 30 Oct 2017.

5. Dong BQ, Yang J, Wang XY, Gong J, von Seidlein L, Wang ML, et al. Trends and disease burden of enteric fever in Guangxi province, China, 1994-2004. Bull World Health Organ. 2010;88:689-96.
6. Yao GH, Tang GP, Tian KC, Zhang J, Sun JL, Wang ZJ, et al. Fever monitoring program in areas with high incidence of typhoid and paratyphoid fever in Guizhou province. Chinese J Epidemiology. 2013;34:254-8 (Article in Chinese).

7. World Health Organization. Human infection with avian influenza a(H7N9) virus - China; March 11, 2015. Available at http://www.who.int/csr/don/11march-2015-avian-influenza-china/en/. Last accessed on 6 Dec 2016.

8. World Health Organization. Human infection with avian influenza a(H7N9) virus - update; February 1, 2014. Available at http://www.who.int/csr/don/ 2014_02_01/en/. Last accessed on 6 Dec 2016.

9. World Health Organization. Laboratory Assessment Tool. Annex 2: Laboratory Assessment Tool / Facility Questionnaire. Available at http://www.who.int/ihr/ publications/Annex__en.xls?ua=1. Last accessed on 2 Dec 2016.

10. Huang $S$, X J J, Deng XL, Wang ZX, Liu ZY, et al. Investigation and strategies on current situation of biological safety management in clinical laboratory of medical institutions in Guizhou province. Guizhou Medical J. 2010:34: 362-5 (Article in Chinese).

11. National People's Congress Standing Committee. The Law of the People's Republic of China on the Prevention and Treatment of Infectious Diseases. Available at http://www.nhc.gov.cn/xxgk/pages/viewdocument. jsp?dispatchDate=\&staticUrl=/zwgkzt/pfl/200804/ bd785fe5acb74ec994d18df71246b521.shtml. Accessed 30 Oct 2017.

12. Wang $\mathrm{R}$, Ni D, Feng Z, Meng L. Infectious disease control laboratory network in China. Chin J Public Health. 2012;28:1258-61 (Article in Chinese).

13. Shuai $Y$. The influence of new medical reform on the financial management of primary health institutions. The Medical Forum. 2013;17(22):2971-3 (Article in Chinese).

14. World Health Organization. Laboratory Quality Stepwise Implementation tool. Available at https://extranet.who.int/lasi/node/338. Last accessed on 2 Dec 2016.

15. The General Office of the Ministry of Health and General Office of the National Development and Reform Commission of P.R. China. Guidance on the Development of Laboratories for Disease Prevention and Control Centers at the provincial, prefectural and county levels ([2004]108) (Guidance in Chinese). Available at http://www.dyscdc.com/main_data_disp. asp?id=75. Last accessed on 2 Dec 2016.

16. World Health Organization.IHR (2005) Monitoring and evaluation framework-joint external evaluation tool (JEE tool). Available at http://www. who.int/ihr/publications/WHO_HSE_GCR_2016_2/en/. Last accessed on 29 Sept 2017.

\section{Ready to submit your research? Choose BMC and benefit from:}

- fast, convenient online submission

- thorough peer review by experienced researchers in your field

- rapid publication on acceptance

- support for research data, including large and complex data types

- gold Open Access which fosters wider collaboration and increased citations

- maximum visibility for your research: over $100 \mathrm{M}$ website views per year

At $\mathrm{BMC}$, research is always in progress.

Learn more biomedcentral.com/submissions 